\title{
The effect of strain and age on the mechanical properties of rat Achilles tendons
}

\author{
Emily C. Vafek \\ Johannes F. Plate \\ Eric Friedman \\ Sandeep Mannava \\ Aaron T. Scott \\ Kerry A. Danelson
}

Department of Orthopaedic Surgery, Wake Forest

School of Medicine, Winston-Salem, USA

Corresponding author:

Kerry A. Danelson

Department of Orthopaedic Surgery,

Wake Forest School of Medicine

Winston-Salem,

NC 27103, USA

E-mail:kdanelso@wakehealth.edu

\section{Summary}

Background: Achilles tendon (AT) ruptures are common in the middle age population; however, the pathophysiology and influence of age on AT ruptures is not fully understood. This study evaluates the effect and interactions between, strain and age on the in vitro biomechanical properties of ATs.

Methods: Bilateral ATs were harvested from 17 young ( 8 months) and 14 middle-aged (24 months) rats and underwent stress-relaxation using Fung's quasilinear viscoelastic (QLV) modeling and load-to-failure testing.

Results: The initial viscoelastic response (parameter B) in middle-age animals was dependent on the amount of strain applied to the tendon and was significantly increased in middle-aged animals at higher strain. Higher strain in older animals led to a prolonged relaxation time (parameter tau 2). There was a trend toward an increased magnitude of the relaxation response (parameter C) at higher strain in the middle-aged animals. Middle-aged animals had a significantly lower mean stress at ultimate failure $(p=0.01)$, while Young's modulus was similar in both groups ( $p=0.46)$.

Conclusions: The passive biomechanical proper- ties of the rat AT change with age and the influence stress-relaxation response of the AT, thereby possibly predisposing the AT of older animals to fail at lower loads compared to younger animals.

Level of evidence: Not applicable, this is a basic science study.

KEY WORDS: aging, fung, quasilinear viscoelastic, Achilles tendon rupture.

\section{Introduction}

Achilles tendon (AT) ruptures occur with an average annual incidence of 7 per every 100,000 people and occur during recreational sports in $80 \%$ of cases ${ }^{1,2}$. These injuries have been shown to be more prevalent in middle-aged men who participate in weekend sporting activities ("weekend warriors"), but the underlying cause remains elusive ${ }^{1,3}$. The primary mechanism of injury has been described as a sudden stretch of the AT during eccentric gastrocnemius muscle contraction ${ }^{4,5}$. The influence of functional, molecular, and histological changes during aging on the biomechanical properties of tendons and ligaments has been assessed in various animal and human studies ${ }^{6-16}$. However, findings in human and animal studies have been equivocal and controversy remains regarding the pathophysiology of AT ruptures in the middle-aged population. While some Authors found decreased strain of the AT during aging ${ }^{11}$, others found increased strain with aging compared to young volunteers ${ }^{12}$. In addition to AT rupture, previous studies have documented changes in mechanical properties with age. Using ultrasound, Waugh et al. quantified an increase in stiffness of the AT with age (from 5 to 35 years) ${ }^{17}$.

Previous studies have utilized a validated Fisher 344 $X$ Brown-Norway F1 hybrid rat model of aging from the National Institute on Aging (NIA) of the National Institute of Health (NIH) to assess underlying changes in biomechanical properties during aging that may predispose middle-aged individuals to AT ruptures using Fung's quasilinear viscoelastic (QLV) modeling ${ }^{18-20}$. QLV parameters A and B characterize the linear and non-linear scaling of the instantaneous elastic response; parameter $\mathrm{C}$ the relaxation scaling, and $t_{1}$ and $t_{2}$ the time-dependent viscous relaxation response ${ }^{18,21-23}$. Previous studies using Fung's QLV to assess stress-relaxation response in this animal 
model revealed that older animals have an increased slope of the instantaneous elastic response (product of parameter A and B), a decreased relaxation response (parameter $\mathrm{C}$ ) and increased stiffness of the Achilles-gastrocnemius muscle-tendon unit in vi$v o^{18,23}$. The findings of this study suggested that with increasing strain, maximum stress may be reached earlier in middle-aged rats compared to young rats. This increase in strain may lead to a higher risk of rupture given the most common injury mechanism as described above. However, strain rate was not examined during these experiments and no load to failure analysis was performed.

The purpose of this study was to evaluate the effect of, and potential interactions between, strain and age on the in vitro biomechanical properties of Achilles tendons in a rat model of aging from the National Institute on Aging. Fung's QLV analysis was used to assess the load-relaxation behavior to two different strains in young and middle aged animals with the hypothesis that aging leads to a decreased stress-relaxation response dependent on strain. Load-to-failure analysis was performed to determine the effect of aging on ultimate failure behavior in these middle-aged rats. With increasing age of the population and higher activity levels, further understanding of the pathomechanism of musculoskeletal injuries will be necessary for the construction of protocols to reduce the incidence of Achilles tendon ruptures in the middle-aged human population.

\section{Materials and methods}

Bilateral Achilles tendons were harvested from 17 young ( 8 months old) and 14 middle-aged (24 months old) Fisher $344 \mathrm{X}$ Brown-Norway F1 hybrid rats from the NIA of the NIH. Animals were part of an unrelated study assessing the influence of aging of the rotator cuff. Specimens represented human age of approximately 18 and 53 years ${ }^{24}$. The AT was dissected from the gastrocnemius muscle while preserving the calcaneal attachment. The leg of the rat was first amputated at the knee and the AT identified. The anterior and lateral compartments were dissected. The calcaneus was detached and a small portion of the soleus/gastrocnemius retained. The clamp was secured to the AT itself during experiment in order to eliminate any cofounding artifacts from muscle tissue. Specimens were placed in saline soaked gauze and frozen at $-20^{\circ} \mathrm{F}^{25}$. Specimens were retrieved for testing and thawed slowly over a twentyfour hour period. Young and middle-aged specimens were assigned to either undergo QLV testing or load to failure testing. Preliminary testing showed that QLV testing affected the mechanical properties of the tendon so these two tests were done separately. The samples were randomized into the different test and strain rate groups; however, the increasing strain QLV tests were always conducted in the same order to prevent damage to the sample. The strain rate for the load to failure and the highest QLV test was selected as 10\%/s because this is representative of activities such as walking and it has been used in previous Achilles tendon test configurations ${ }^{26}$. Mechanical property testing was performed on a materials testing machine using aluminum clamps at both ends of the tendon (Instron 4201, Norwood, MA, USA). The tendon was assumed to be an ellipse and the major and minor axis were measured before clamping. The main axes were defined by the orientation of the tendon within the grips. The calcaneus was gripped along the medial and lateral edge so the tendon was pulled in an approximately anatomic orientation. The major axis was measured along the anterior/posterior axis and the minor axis was along the medial/lateral axis. The reproducibility of these measurements was verified during protocol development. The major axis was monitored over the course of the test using a laser measurement system (IG-028, Keyence, Itasca, IL). Given the small variations recorded, the area was assumed to be constant for stress calculations, similar to other previous test configurations ${ }^{27}$. The specimen length was measured at a preload of $1.5 \mathrm{~N}$ prior to testing. This is a slight increase over previously published tests; however, the slightly higher pre-load resulted in fewer instances of the tendon slipping from the grips during protocol development ${ }^{18,27}$. Additionally, it is well below $5 \%$ of the ultimate failure load. In addition, specimens were sprayed with saline every minute in order to prevent desiccation. There were two testing protocols: QLV and load-to-failure. Displacement of the tendon was measured both by the absolute displacement of the upper clamp and by using high speed video camera (HHC X3, Dynamic Imaging, Mt. Laurel, NJ, USA) to track ink dots placed along the length of the tendon. The high speed video camera data was also used to determine if the tendon slipped relative to the clamp. Tests in which the tendon slipped were not counted in the results. Stress and strain were calculated from the previously described measurements. Stress $(\delta)$ was calculated by dividing force $(F)$ by area $(A)$ from the initial calculation, i.e. $\delta=F / A^{26}$. Engineering strain $(\varepsilon)$ was calculated by dividing the change in length $(\Delta \mathrm{L})$ of the tendon by the initial length $\left(L_{\circ}\right)$, i.e. $e=\Delta L /$ $\mathrm{L}_{0}{ }^{26}$. The size of the tendons was not normalized prior to testing because these equations were able to account for size variations between the animals. The study was approved by the Institutional Animal Care and Use Committee. This study was conducted in accordance with the ethical standards required by this Journal and outlined by Padulo et al. ${ }^{28}$.

\section{QLV protocol}

QLV testing and analysis was performed similar to previous reports ${ }^{18,22,23,29}$. A $100 \mathrm{~N}$ load cell was installed into the testing machine and the tendon was clamped at $20 \mathrm{psi}$. The air pressure was then increased to $40 \mathrm{psi}$ in order to prevent slippage. A preload of $1.0 \mathrm{~N}$ was set at $0.01 \mathrm{~N} / \mathrm{s}$. Pre-cycling was done for 10 cycles of loading from $1.0 \mathrm{~N}$ to $2.0 \mathrm{~N}$ at $0.1 \mathrm{~N} / \mathrm{s}$. There was a rest period of 300 seconds at $0.1 \mathrm{~N}$. Stress-relaxation testing commenced with $5 \%$ 
strain, $10 \%$ strain, at a rate of $10 \% / \mathrm{s}$. After tensile loading, the tendon was held for 600 seconds before translating back the same distance it was extended. A 300 second rest period was adhered to after the first stress-relaxation cycle.

\section{Load-to-failure protocol}

The tendon was clamped into the testing machine at $20 \mathrm{psi}$, and then the air pressure was increased to 40 psi in order to keep the tendon secure so there would be no slipping during testing. A preload of $1.0 \mathrm{~N}$ was set at $0.01 \mathrm{~N} / \mathrm{s}$. Pre-cycling was done for 10 cycles of loading from $1.0 \mathrm{~N}$ to $2.0 \mathrm{~N}$ at $0.1 \mathrm{~N} / \mathrm{s}$ in order for all of the tendon's fibers to align. Then, there was a rest period of 300 seconds at $0.1 \mathrm{~N}$. Using a $500 \mathrm{~N}$ load cell, the load-to-failure testing commenced at a strain rate of $10 \% / \mathrm{s}$. Testing concluded once a drop in $75 \%$ of the maximum recorded force was reached.

\section{Statistical analysis}

Young's modulus was obtained by performing a linear regression of the elastic region of the stress versus strain plot. The linear region was identified through visual inspection and the slope was calculated using a straight line estimation of two points within this region. All values are expressed as mean \pm standard error of the mean (SEM) and statistical analysis performed using Prism5 (GraphPad Software, La Jolla, CA, USA). Statistical comparison of failure and QLV testing within animal age groups was performed using paired Student t-tests with alpha 0.05. QLV data comparisons between animal age groups were performed using ANOVA with Sidak's multiple comparison test with alpha 0.05 .

\section{Results}

A total of 40 tendons were used for load-relaxation testing (23 young, 17 old). QLV data for 1 tendon from an old animal had to be excluded due to clamp slippage. QLV analysis in young animals revealed a similar viscoelastic response to 5 and $10 \%$ strain (Tab. I). The initial viscoelastic response (parameter B) in middle-age animals was dependent on the amount of strain applied to the tendon and was significantly increased in middle-aged animals at higher strain (Tab. II). Higher strain in older animals led to a prolonged relaxation time (parameter tau 2). There was a trend toward an increased magnitude of the relaxation response (parameter $\mathrm{C}$ ) at higher strain in the middle-aged animals.

The relative change in QLV response of young and middle-aged animals was compared using ANOVA (Tab. III) The relative change in QLV response was similar between young and middle-aged animals. The product of $A$ and $B$ (initial slope of the elastic response) increased by a mean $79 \%$ in young animals between the two strains applied compared with a mean of $-2 \%$ in old animals.

A total of 31 tendons underwent load-to-failure testing (11 young, 20 old). Failure occurred in the midsub- stance of the tendon in all cases, similar to rupture patterns seen clinically ${ }^{30}$. Middle-aged animals had a significantly lower mean stress at ultimate failure $(p=0.01)$ compared with young (Tab. IV, Fig. 1). The Young's modulus was found to similar in the two groups $(p=0.46)$ (Tab. IV, Fig. 1). While there is overlap between the responses of the two groups and similar slopes (Young's modulus), the younger group demonstrated higher ultimate load to failure.

\section{Discussion}

The findings of this study revealed that aging influences the QLV response of the Achilles tendon to increased strain in an animal model. The AT in middleaged animals failed at a lower ultimate load compared with young animals. Middle-aged animals were found to have a delayed QLV response to higher strain compared to young animals. Based on the most common injury mechanism for AT ruptures, these findings suggest that increased strain simulating eccentric activation of the AT-gastrocnemius muscle tendon unit ${ }^{5}$ leads to decreased accommodation to an applied load in middle-aged animals. The decreased relaxation response in middle-aged animals may contribute to failure at lower loads. These findings depict one possible aspect of the underlying pathophysiology of AT ruptures in the middle aged population.

There is a limited number of basic science studies utilizing the NIA rodent model of aging to study agerelated changes on the musculoskeletal system that may influence common orthopaedic injuries. The NIA Fisher $344 \mathrm{X}$ Brown-Norway F1 hybrid rat model exhibits age-related changes in various organ systems including sarcopenia, decreased muscle mass and function similar to humans ${ }^{18-20,31,32}$. Various molecular and structural changes of tendon with aging have been proposed that can influence biomechanical material properties of tendon and muscle tissue including decreased collagen content and disorganization and decreased muscle and tendon healing response ${ }^{33-36}$. The findings of this study using the validated model of aging of the musculoskeletal system showed that aging alone influences the ultimate load to failure of the AT. The ATs in middle-aged animals were found to fail at approximately $46 \%$ lower stress compared to young animals. This finding concurs with previous reports of anterior cruciate ligament and patellar tendon experiments that revealed failure at lower stress with increasing age ${ }^{37,38}$.

QLV analysis in the current study revealed that increased strain in middle-aged animals led to a decreased relaxation. This finding is similar to a previous study of the entire AT-gastrocnemius muscle tendon unit in a similar aging rat model in vivo ${ }^{18}$. The previous study revealed a difference in QLV parameter $\mathrm{C}$ which is associated with decreased muscle relaxation toward its normal state ${ }^{18}$, implicating a higher risk for tendon microtears with repetitive loading ${ }^{3,39}$. However, strain was not utilized as an independent 
Table I. QLV analysis of young animals.

\begin{tabular}{llll}
\hline QLV parameter & $\mathbf{5 \%}$ strain & $\mathbf{1 0 \%}$ strain & p-values \\
\hline A & $0.112 \pm 0.43$ & $0.022 \pm 0.04$ & 0.387 \\
B & $4.667 \pm 1.55$ & $6.501 \pm 3.40$ & 0.128 \\
C & $0.143 \pm 0.06$ & $0.191 \pm 0.19$ & 0.209 \\
Tau 1 & $0.143 \pm 0.18$ & $0.160 \pm 0.26$ & 0.698 \\
Tau 2 & $318.2 \pm 245.3$ & $154.0 \pm 134.3$ & $0.018^{*}$ \\
A x B & $0.045 \pm 0.04$ & $0.069 \pm 0.09$ & 0.073 \\
\hline
\end{tabular}

QLV, quasilinear viscoelastic; *a p-values less than 0.05 was considered significant.

Table II. QLV analysis of middle-aged animals.

\begin{tabular}{llll}
\hline QLV parameter & 5\% strain & $\mathbf{1 0 \%}$ strain & p-values \\
\hline A & $0.164 \pm 0.58$ & $0.007 \pm 0.01$ & 0.317 \\
B & $3.505 \pm 7.74$ & $7.519 \pm 1.93$ & $<0.001^{*}$ \\
C & $0.129 \pm 0.05$ & $0.165 \pm 0.08$ & 0.067 \\
Tau 1 & $0.2197 \pm 0.27$ & $0.120 \pm 0.18$ & 0.106 \\
Tau 2 & $421.0 \pm 213.5$ & $143.0 \pm 72.76$ & $<0.001^{*}$ \\
A x B & $0.040 \pm 0.03$ & $0.039 \pm 0.5$ & 0.951 \\
\hline
\end{tabular}

$1 \mathrm{QLV}$, quasilinear viscoelastic; *a p-values less than 0.05 was considered significant.

Table III. Comparison of the mean and confidence interval for the failure stress and Young's modulus between the young and middle-age group.

\begin{tabular}{llll}
\hline Age & $\mathbf{N}$ & Failure Stress $\left(\mathbf{N} / \mathbf{m m}^{2}\right)$ & $\mathrm{E}\left(\mathrm{N} / \mathrm{mm}^{2}\right)$ \\
\hline Young & 11 & $7.72 \pm 3.87$ & $482.27 \pm 380.05$ \\
Middle-aged & 20 & $4.14 \pm 2.24$ & $503.39 \pm 546.48$ \\
P-value & & $\mathrm{p}<0.01$ & $\mathrm{p}=0.46$ \\
\hline
\end{tabular}

Table IV. Comparison of mean relative change (in percent) in QLV response to strain between young and middleaged animals.

\begin{tabular}{llll}
\hline QLV parameter & Young & Middle-aged & p-values \\
\hline A & $130 \% \pm 82 \%$ & $-14 \% \pm 33 \%$ & NS \\
B & $187 \% \pm 150 \%$ & $167 \% \pm 57 \%$ & NS \\
C & $30 \% \pm 14 \%$ & $36 \% \pm 15 \%$ & NS \\
Tau 1 & $53 \% \pm 70 \%$ & $-23 \% \pm 9 \%$ & NS \\
Tau 2 & $-27 \% \pm 57 \%$ & $-61 \% \pm 5 \%$ & NS \\
A x B & $79 \% \pm 34 \%$ & $-2 \% \pm 29 \%$ & NS \\
\hline
\end{tabular}

QLV, quasilinear viscoelastic; NS, not significant based on $p$-values greater than 0.05 during ANOVA testing. 


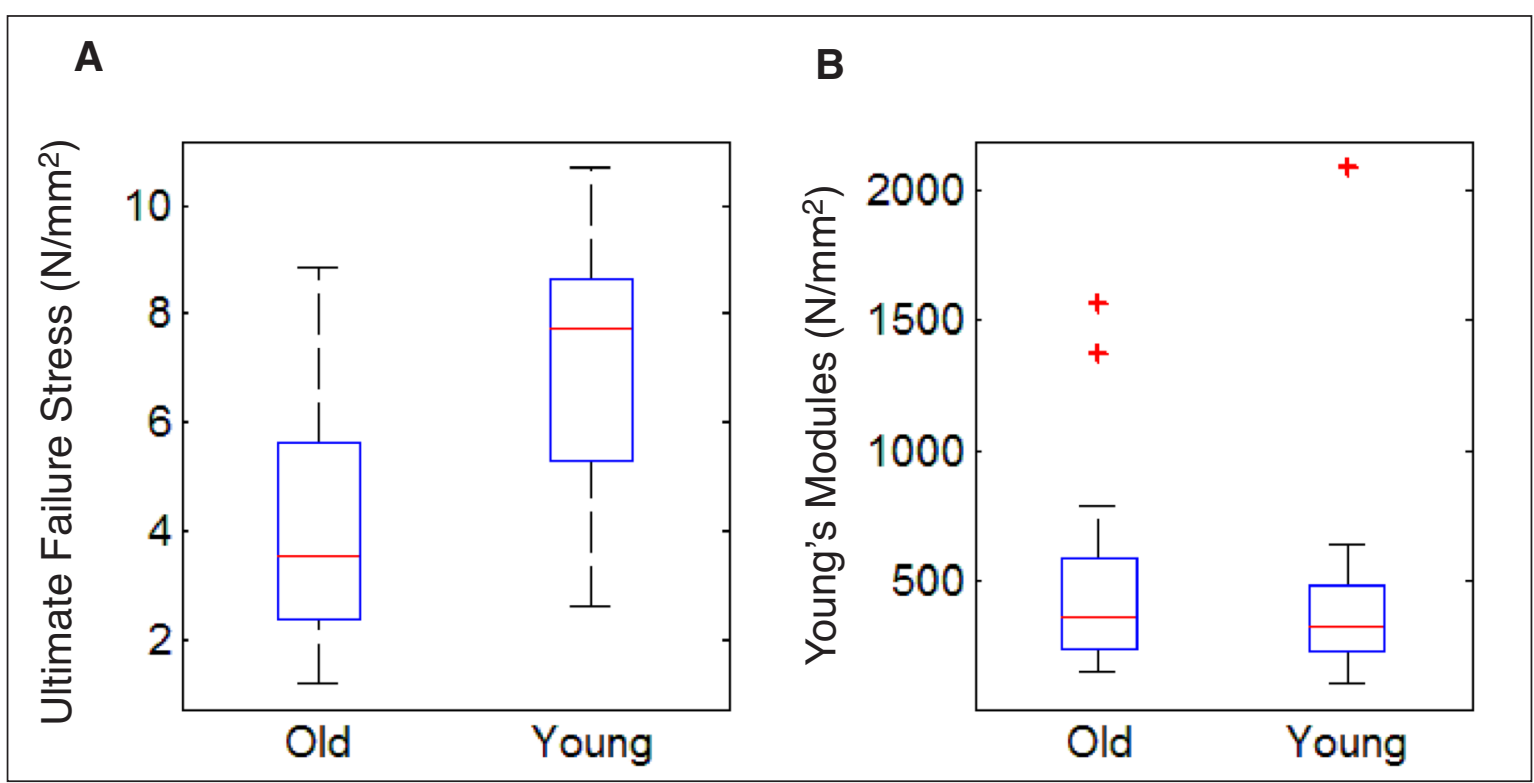

Figure 1. Box plots of the comparison of Young's modulus and ultimate failure stress demonstrating the similarity in the modulus and the variation in failure stress.

parameter in that study which appears to be important based on the most common AT injury mechanism. The current study revealed a significant increase in QLV parameter B, the non-linear scaling of the instantaneous elastic response, with increased strain in middle aged animals. This implies that with increased strain there is less relaxation and accommodation of the AT with aging, given that QLV parameters were similar in young animals regardless of strain. However, comparison of the relative change in QLV response to increased strain between the two age groups was statistically similar. The product of $A$ and $B$ increased by a mean of $80 \%$ in young animals compared with a mean decreased of $2 \%$ in middleaged animals. This trend toward a decrease in the slope of the instantaneous elastic response in middleaged animals may predispose older patients to AT failure with high loading forces and high strain of the AT seen in certain sports (basketball, tennis, soccer). The limitations of this study are related to the use of an animal model to elucidate the biomechanical response of the human musculoskeletal system to aging of the AT. The size of the rat AT may not allow for accurate scaling to the human AT. Experiments were performed on the AT in isolation. While previous studies have analyzed the AT gastrocnemius muscle-tendon unit, this study focused on the impact of aging on biomechanical properties of the tendon only. Therefore, it needs to be recognized that the findings of the ultimate load to failure analysis relate to the AT. However, the most common site of injury in middle-aged patients is in the mid-substance of the tendon which was analyzed in this report. The number of animals was limited by the availability of the animal model which may have influenced the ability of the study to reveal statistical differences in the QLV response between age groups. Finally, the age properties were only assessed at two time points instead of a continuous range. These time points were selected based on the distribution of Achilles tendon rupture in middleaged athletes. Future studies should expand the range of ages tested.

In conclusion, this study provides evidence that passive mechanical properties of the AT change with age. Middle-aged ATs showed significantly lower failure stress. The QLV response of the AT to increased strain revealed a trend toward a decreased slope of the initial elastic response, possibly predisposing the AT of older individuals to fail at lower loads compared to younger individuals. However, further studies are needed to identify underlying histological or molecular changes that occur inside the tendon substance.

\section{Conflict of interest}

No other relationships, conditions, or circumstances present potential conflict of interest.

\section{References}

1. Leppilahti J, J Puranen, S Orava, Incidence of Achilles tendon rupture. Acta Orthop Scand. 1996:67(3):277-279.

2. Suchak $A A$, et al. The incidence of Achilles tendon ruptures in Edmonton, Canada. Foot Ankle Int. 2005;26(11):932-936.

3. Jarvinen TA, et al. Achilles tendon injuries. Curr Opin Rheumatol. 2001;13(2):150-155.

4. Sharma $P, N$ Maffulli. Tendon injury and tendinopathy: healing and repair. J Bone Joint Surg Am. 2005;87(1):187-202.

5. Lin R, G Chang, L Chang. Biomechanical properties of muscle-tendon unit under high-speed passive stretch. Clin Biomech (Bristol, Avon). 1999;14(6):412-417.

6. Clemmer J, et al. A mechanistic study for strain rate sensitivity of rabbit patellar tendon. J Biomech. 2010:43(14):2785-2791. 
7. Vogel HG. Age dependence of mechanical properties of rat tail tendons (hysteresis experiments). Aktuelle Gerontol. 1983;13 (1):22-27.

8. LaCroix AS, et al. Effect of age and exercise on the viscoelastic properties of rat tail tendon. Ann Biomed Eng. 2013;41(6): 1120-1128.

9. Pioletti DP, LR Rakotomanana, PF Leyvraz. Strain rate effect on the mechanical behavior of the anterior cruciate ligamentbone complex. Med Eng Phys. 1999;21(2):95-100.

10. Haut RC. Age-dependent influence of strain rate on the tensile failure of rat-tail tendon. J Biomech Eng. 1983;105(3):296-299.

11. Kubo K, et al. Age-related differences in the properties of the plantar flexor muscles and tendons. Med Sci Sports Exerc. 2007;39(3):541-547.

12. Onambele GL, MV Narici, CN Maganaris. Calf muscle-tendon properties and postural balance in old age. J Appl Physiol. 2006;100(6):2048-056.

13. Karamanidis K, A Arampatzis. Mechanical and morphological properties of human quadriceps femoris and triceps surae muscle-tendon unit in relation to aging and running. $J$ Biomech. 2006;39(3):406-417.

14. Dressler MR, et al. A potential mechanism for age-related declines in patellar tendon biomechanics. J Orthop Res. 2002;20 (6):1315-1322.

15. Goh KL, et al. Ageing changes in the tensile properties of tendons: influence of collagen fibril volume fraction. J Biomech Eng. 2008;130(2):021011.

16. Grasa J, et al. Variations in tendon stiffness due to diets with different glycotoxins affect mechanical properties in the muscle-tendon unit. Ann Biomed Eng. 2013;41(3):488-496.

17. Waugh $\mathrm{CM}$, et al. Age-related changes in mechanical properties of the Achilles tendon. J Anat. 2012;220(2):144-155.

18. Plate JF, et al. Normal aging alters in vivo passive biomechanical response of the rat gastrocnemius-Achilles muscle-tendon unit. J Biomech. 2013;46(3):450-455.

19. Betik AC, et al. Exercise training in late middle-aged male Fischer 344 x Brown Norway F1-hybrid rats improves skeletal muscle aerobic function. Experimental Physiology. 2008;93 (7):863-871.

20. Rice KM, et al. The Fischer 344/NNiaHSd X Brown Norway/ $\mathrm{BiNia}$ is a better model of sarcopenia than the Fischer 344/NNiaHSd: a comparative analysis of muscle mass and contractile properties in aging male rat models. Biogerontology. 2005;6(5):335-343.

21. Fung YC. Elasticity of soft tissues in simple elongation. Am J Physiol. 1967;213(6):1532-1544.

22. Fung YC. Stress-strain-history relations of soft tissues in simple elongation. In: Biomechanics: Its Foundations and Objectives, edited by YC Fung, N Perrone, M Anliker. San Diego: Prentice-Hall. 1972:181-208.

23. Abramowitch SD, et al. A comparison of the quasi-static mechanical and non-linear viscoelastic properties of the human semitendinosus and gracilis tendons. Clinical Biomechanics.
2010;25(4):325-331.

24. Quinn R. Comparing rat's to human's age: how old is my rat in people years? Nutrition. 2005;21(6):775-777.

25. Freedman BR, et al. Biomechanical and structural response of healing Achilles tendon to fatigue loading following acute injury. J Biomech. 2014;47(9):2028-2034.

26. Wren TA, et al. Mechanical properties of the human Achilles tendon. Clin Biomech (Bristol, Avon). 2001;16(3):245-251.

27. Boivin GP, et al. Biomechanical properties and histology of $\mathrm{db} / \mathrm{db}$ diabetic mouse Achilles tendon. Muscles Ligaments Tendons J. 2014;4(3):280-284.

28. Padulo J, Oliva F, Frizziero A, Maffulli N. Muscles, Ligaments and Tendons Journal - Basic principles and recommendations in clinical and field science research: 2016 update. MLTJ. 2016;6(1):1-5.

29. Mannava S, et al. Contributions of Neural Tone to In Vivo Passive Muscle - Tendon Unit Biomechanical Properties in a Rat Rotator Cuff Animal Model. Ann Biomed Eng. 2011;39(7): 1914-1924.

30. Longo UG, M Ronga, N Maffulli. Acute ruptures of the Achilles tendon. Sports Med Arthrosc. 2009;17(2):127-138.

31. Hagen JL, et al. Skeletal muscle aging in F344BN F1-hybrid rats: I. Mitochondrial dysfunction contributes to the age-associated reduction in VO2max. Journals of Gerontology. Series A, Biological Sciences and Medical Sciences. 2004;59(11): 1099-1110.

32. Lipman RD, et al. Pathologic characterization of brown Norway, brown Norway x Fischer 344, and Fischer 344 x brown Norway rats with relation to age. Journals of Gerontology. Series A, Biological Sciences and Medical Sciences. 1996;51(1): B54-59.

33. Delbono O. Molecular mechanisms and therapeutics of the deficit in specific force in ageing skeletal muscle. Biogerontology. 2002;3(5):265-270.

34. Reeves ND, MV Narici, CN Maganaris. Myotendinous plasticity to ageing and resistance exercise in humans. Experimental Physiology. 2006;91(3):483-498.

35. Plate JF, et al. Age-related changes affect rat rotator cuff muscle function. J Shoulder Elbow Surg. 2014;23(1):91-98.

36. Plate JF, et al. Advanced age diminishes tendon-to-bone healing in a rat model of rotator cuff repair. Am J Sports Med. 2014; 42(4):859-868.

37. Noyes FR, JL DeLucas, PJ Torvik. Biomechanics of anterior cruciate ligament failure: an analysis of strain-rate sensitivity and mechanisms of failure in primates. J Bone Joint Surg Am. 1974;56(2):236-253.

38. Johnson GA, et al. Tensile and viscoelastic properties of human patellar tendon. J Orthop Res. 1994;12(6):796-803.

39. Maffulli N, et al. Tenocytes from ruptured and tendinopathic Achilles tendons produce greater quantities of type III collagen than tenocytes from normal Achilles tendons. An in vitro model of human tendon healing. Am J Sports Med. 2000;28(4):499505. 